\title{
Utilising Mobile Phone RSSI Metric for Human Activity Detection
}

\author{
John Doyle ${ }^{\dagger}$, Ronan Farrell, Seán McLoone, Tim McCarthy, Muhammad Tahir and \\ Peter Hung
}

\author{
Institute of Microelectronics and Wireless Systems \\ National University of Ireland Maynooth \\ Ireland \\ E-mail: ${ }^{\dagger}$ jcdoyle@eeng.nuim.ie
}

\begin{abstract}
Recent research into urban analysis through the use of mobile device usage statistics has presented a need for the collection of this data independently from mobile network operators. In this paper we propose that cumulative received signal strength indications (RSSI) for overall mobile device transmissions in an area may provide such independent information. A process for the detection of high density areas within the RSSI temporal data set will be demonstrated. Finally, future applications for this collection method are discussed and we highlight its potential to complement traditional metric analysis techniques, for the representation of intensity of urban and local activities and their evolution through time and space.
\end{abstract}

Keywords - Mobile communications, RSSI, Erlang, urban analysis, human activity, geographical mapping, temporal analysis

\section{INTRODUCTION}

Techniques for sensing the flow of people are creating new means for observing the dynamics within our local environments. In many developed countries mobile phone devices are starting to outnumber people. In Ireland alone the number of mobile phones in 2007 numbered 5.3 million [1] while the human population numbered 4.3 million [2]. With the aid of such devices, activities that once required a fixed location and connection can now be achieved with higher flexibility. This enables users to act and communicate more freely making the collection of human flow patterns more and more difficult and costly.

However, as mobile devices are generally carried by people and usually remain switched on, the detection of their usage is indicative of the presence of some number of people in that approximate area. Therefore, by monitoring the mobile spectrum band we have an opportunity to observe and interpret in real-time, the ubiquitous connectivity between people and the environments they pass through [3].

A common approach to extract such mobile device activity measurements is to focus on the use of mobile operator statistical data. This data is then used to model the dynamics and behaviours in the area of interest. However, this method relies on having the cooperation of network operators, without which no usage data would exist.

The usage statistic commonly employed in these mapping applications is a measure of network bandwidth used. Typically this is collected at a base station in a mobile operator's network, or by use of special software added to a person's mobile phone. The metric by which this activity is measured is known as an Erlang [4]. An Erlang is one person-hour of phone use, which could represent one person talking for an hour, two people talking for half an hour each, 30 people each speaking for two minutes, and so on. This method was valuable in the past due to the restricted nature of mobile telecommunications which were fundamentally voice-only networks. Modern networks have an increasingly diverse range of usages which do not linearly correspond to intensity of communication. For instance text messaging uses very little bandwidth though are an increasingly important form of communication.

We propose that as an alternative to monitoring data throughput measurements, the cumulative elec- 
tromagnetic energy in the frequency range of clientside mobile phone transmissions may be used instead. This metric will provide a more modern interpretation of quantity of digital data transferred, whether voice, SMS, or data. It is non-intrusive and has none of the privacy concerns of the previous method and can be easily achieved using well known circuits for Received Signal Strength Indication (RSSI) [5] [6]. By analysing RSSI values over time and space through a collaborative network of sensors, this approach may provide comparable results to network centric mobile device analysis methods and give better spatial resolution. In addition, RSSI measurements are inherently anonymous due to the absence of information decoding. As a result, it is impossible to deduce individual identities or phone messages from the raw data collected and stored in the proposed system, thus avoiding potential ethical issues.

In the rest of this paper, we highlight the use of an energy detecting device to monitor mobile spectrum activity. Section II gives an overview of some related work in this field. Section III and Section IV details the process we propose to extract useful information from the RSSI temporal data set. Section V presents the results of some preliminary work and Section VI suggests the future work to be carried out and conclusions.

\section{BACKGROUND}

This section presents on overview of some related research on the collection and analysis of mobile device usage data. The results can be classified as being based on either mobile landscape mapping or spectrum signal strength collection.

\section{a) Mobile Phone Usage Mapping}

Monitoring mobile phones usage patterns to extract human behavioural patterns has been a topic of growing research interest over the last few years. There are two aspects to this research: data collection at the operator level; and the other through modified mobile phone software.

The first area requires the cooperation of mobile operators to provide data on a macro level of urban areas. Examples of two such projects are the "Mobile Landscapes" [3] project and "Real Time Rome" [7] project. These projects queried network operators for usage statistical information providing them with the information necessary to produce maps of human flow. They highlighted the potential of mobile usage mapping technology in the context of urban planning.

The Mobile Landscapes [3] project collected network usage data in Milan, Italy. When combined with the geographical mapping of cell areas, a graphical representation of the intensity of urban activities and their evolution through space and time was produced. From this they were able to detect events such as national holidays and major sporting events.
The Real Time Rome project [7] was the first example of an urban-wide real time monitoring system that collects and processes data provided by telecommunications networks and transportation systems. It used location data from mobile phone subscribers provided by Telecom Italia, public buses by a local transport company Atac and taxis run by the cooperative Samarcanda.

There are however difficulties with this approach. There are certain legal and privacy issues that prevent operators delivering such information to outside researchers. In addition, even with best efforts, there is no guarantee that data from theses sources is always available, complete or accurate. A more fundamental issue arises as mobile network topologies become more dynamic and are optimised throughout the day and temporary towers are increasingly used for special events. This adds a level of uncertainty into these fixed point measurements. In addition, the spatial resolution of the usage statistics is dependent on the operator's network topology which may be very limited in lower populated areas.

The other approach, based on mobile handsets, addresses this issue by placing embedded software application on the mobile devices to log data. MIT's "Reality Mining" project illustrated that it was possible to extract common behavioural patterns from the activities of 94 subjects [8]. The subjects were issued with mobile phones pre-installed with several pieces of software that record and sent research data on call logs, Bluetooth devices in proximity, cell tower IDs, application usage, and phone status. This yields valuable, person specific results but the solution may not scale to the large numbers needed to represent an urban population.

\section{b) Spectrum RSSI Collection}

Measuring signal strengths is a common technique in wireless communications and RSSI devices can be easily purchased or built. Due to its ready availability, it has been considered in the past as a sensing parameter and a number of applications have provided insight into its usefulness. Both Wu et al. [9] and Stoyanova et al. [10] describe the key issues which affect RSSI accuracy. They are summarised as:

- The orientation of the antenna

- Transceiver variation

- Multipath fading and changes in environment

Cognitive radio systems [11] [12] have become increasingly viable and signal strength measurement is a key element in the detection of primary user spectral occupancy. To improve performance, they have explored a number of techniques that can be used to address these issues, in particular collaborative sensing between multiple RSSI detectors. By cross-correlation 
and signal processing, non-random signals can be detected and analysed. Similar approaches can be applied with existing transmissions to detect usage and extract statistics.

\section{Proposal}

Our proposal is based on the measurement of localised cumulative strength of mobile device emissions through the use of an RSSI sensor. From this collected data we can then extract behavioural patterns of human activity, providing an alternative to the Erlang metric. As an alternative sensing parameter, cumulative received signal strength (RSSI) offers several advantages over network usage data (the Erlang metric);

- RSSI data can be collected without the cooperation of mobile operators

- RSSI, as a metric is independent of modulation type, so RSSI can be used for GSM protocols and $3 \mathrm{G}$ protocols

- RSSI data can provide fine spatial and temporal resolution making it possible to localise events very accurately and quickly

However, signal strength sensing or RSSI measurements from a single source has limitations in terms of spatial accuracy. We propose that these accuracy issues may be mitigated by adopting techniques commonly utilised in cognitive radio research. Firstly, by spatially and temporally weighting each RSSI data point form a sensor with corresponding points from other radios in the geographical area nearby, the RSSI accuracy can be improved [13] [14]. Secondly, modelling the environment with accurate models will help quantify the data and give insight into its behaviour. Thirdly, calibration with respect to base station coverage will reduce effects caused by mobile device transmission power variation. Finally, the spatial sampling topology of the sensor network will be a dominant factor in determining performance, particularly where variable sensor heights are used, thus methods for insuring topology uniformity must be taken into account.

An issue may arise when trying to gauge the RSSI activity to mobile device ratio, namely how to distinguish between the RSSI signal generated by one user near the sensor and several users further away. By having a dense network topology, we can insure that spectral energy readings from each sensor can be localised to some degree. There are several possible solutions to this problem. One solution is to localise activity by a sensor identification process, a technique similar to the cell identification process described in Section II. Here the sensor node with the associated highest RSSI value is deemed to be the coordinate of the activity. This will however offer reduced spacial resolution, thus a more advanced technique which combines multi-sensor information in a effort to accurately locate the mobile devices would be a more suitable approach.

\section{Temporal Weighted Processing}

Various signal processing algorithms can be applied to assist with extracting interesting patterns from measured mobile phone signal strengths. Our approach to date, has focused on a temporal based scheme that identifies time periods with interesting behaviour. One early approach is explained in this section. Its layout is depicted in Fig. 1.

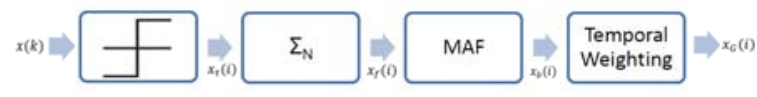

Fig. 1: Post signal processing method performed on raw RSSI data.

The spectral energy was sampled at a rate of $2 \mathrm{kHz}$ (2000 samples per-second) and is denoted by $x(k)$. The signal processing method applied to these samples consists of four stages;

- Stage 1: Detect the presence of a mobile transmission as governed by a threshold $\tau$

$x_{\tau}(k)= \begin{cases}0 & \text { if } x(k)<\tau \\ x(k) & \text { if } x(k) \geq \tau\end{cases}$

where, $\tau$ in this instance is chosen to be $-55 \mathrm{dBm}$, the minimum detectable level of the energy detecting chipset.

- Stage 2: Down sample the data by a factor of $T$, this is done by replacing every block of $T$ samples by its average

$x_{b}(i)=\frac{1}{T} \sum_{k=(i-1) T+1}^{i T} x_{\tau}(k)$

where, $x_{b}(i)$ is the index of the down sampled data set and $T$ is the down sampling factor. Decimation should be application specific. While it can trim down the noise within the data, excessive decimation may reduce the signal of short temporal events, such as text messages.

- Stage 3: Smooth the data using a moving average filter (MAF) of width $(2 W+1)$ samples

$x_{f}(i)=\frac{1}{W} \sum_{p=(i-W)}^{i+W} x_{b}(p)$

where, $x_{f}(i)$ is resulting filtered data set.

- Stage 4: Apply a temporal weighting to each sample using a truncated Gaussian Kernel, that is

$$
x_{G}(i)= \begin{cases}\sum_{p=(i-\phi)}^{i+\phi} x_{f}(p) \theta_{\lambda}[i-p] & \text { if } x_{f}(p)<\gamma \\ \sum_{p=(i-\phi)}^{i+\phi} x_{f}(p) \theta_{\lambda \beta}[i-p] & \text { if } x_{f}(p) \geq \gamma\end{cases}
$$

where, 
$-\theta_{a}[b]=\exp -\left(\frac{b}{a}\right)^{2}$

- $x_{G}(i)$ is the weight associated with a point in time corresponding to the truncated Gaussian Kernel

$-\lambda$ is scalar controlling the spreading factor of the truncated Gaussian Kernel

- $\beta$ is extra spreading factor weight given to those points whose power is over the threshold $\gamma$

$-2 \phi+1$ is the truncated Gaussian Kernel window size

The effect of this stage is to both amplify and smooth the data further. Areas that are temporally close and deemed to have high magnitude, above a threshold $\gamma$, will be further amplified.The effect of which is to highlighting areas of high temporal dense activity. By re-applying this transform to the data set a higher order weighting function can be achieved.

\section{EXPERIMENTAL Work}

\section{a) Experimental Setup}

Our experiments were based on the measurement of localised cumulative strength of mobile device emissions through the use of a RSSI sensor. The equipment used to measure the RSSI intensity was a true power detector from Analog Devices (chip part number AD8362) paired with a single omni-directional GSM 900 antenna. The AD8362 device returns a voltage which linearly corresponds to the RF spectrum power passed through it. It operates with a $65 \mathrm{~dB}$ dynamic range, ranging from $-55 \mathrm{~dB}$ to $10 \mathrm{~dB}$.

The experiments undertaken were as follows;

- Experiment 1: A control test was performed, which consisted of a single text message followed by a single phone call (GSM network). No other phone activity was present at the graphical location while this test was carried out.

- Experiment 2: An experiment was carried out in an uncontrolled manner at the same geographical location as used for Experiment 1. Here normal mobile device activity was observed from the student population over two time instances (A and B).

\section{b) Results}

The results shown here reflect measurements of localised mobile spectrum RSSI through the use of a energy detecting sensor. Each set of results was taken within a building on NUI Maynooth's North Campus. Fig. 2 displays the results form experiment 1 , while Figs. 3 and 4 present the results from experiment 2 .

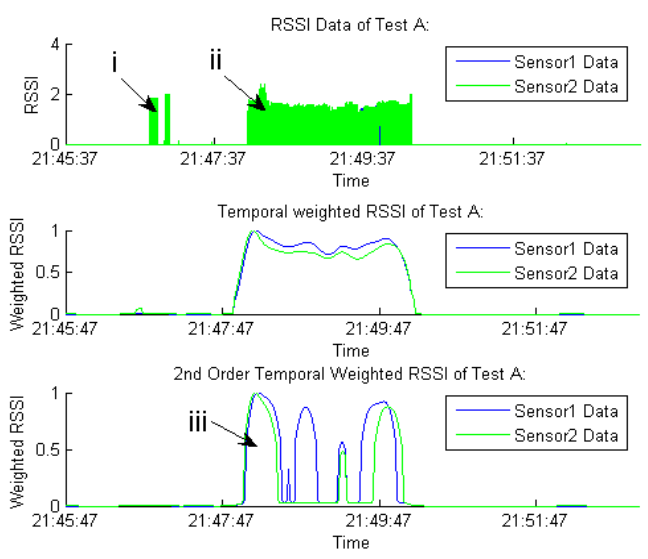

Fig. 2: Controlled experiment consisting of a single phone call and text message; 'i' represents a text message; 'ii' represents a phone call; 'iii' represents patterns identified in the phone call RSSI temporal data.
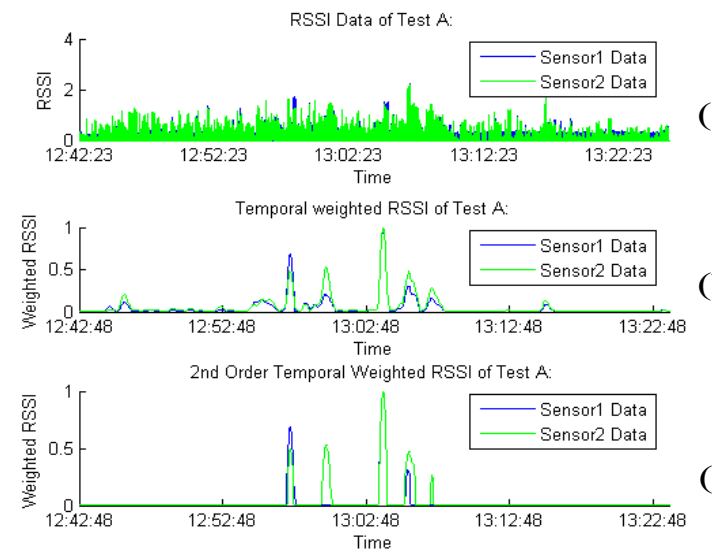

Fig. 3: Normal mobile device activity of the student population at NUI Maynooth, Experiment 2(A). (c) The second order results reflect the passing of the data through the temporal weighting function twice

The results show that high temporal density areas have been identified in the experiments. From examining Fig. 2 we can see the behaviour of a GSM phone call. The initial peak, as indicated by 'iii' in Fig. 2(c), reflects the handshake between the base-station and a mobile phone. The remaining pulses reflect times where the phone user spoke into the device. These events occur because GSM mobile phones only transmit high density bursts when they have to transmit speech during a normal communication (data or voice). This is done to conserve the devices battery.

Experiments performed on a non-controlled environment highlight events occurring close to each hour mark. Theses events relate to times where classes finished and started. In post processing the raw RSSI data, we adopted two approaches. One approach used a single order temporal weighing. These results, which as depicted in Fig. 3(b) and Fig. 4(b), represent the overall density pattern of mobile activity. The second approach, as depicted in Fig. 3(c) and Fig. 4(c), focused 

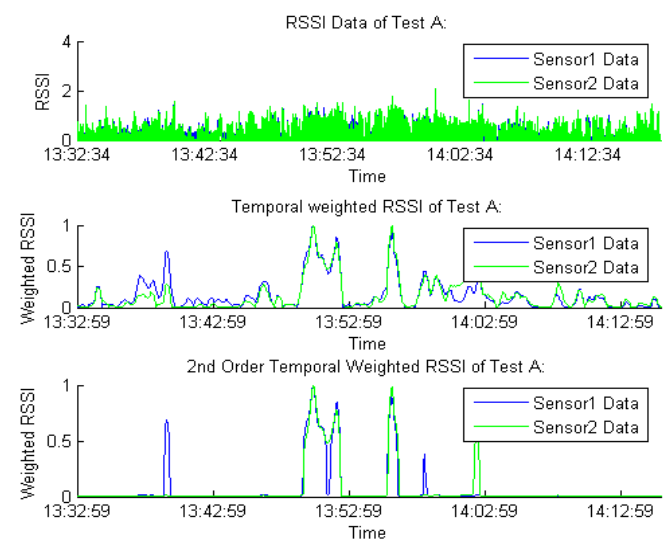

Fig. 4: Normal mobile device activity of the student population at NUI Maynooth, Experiment 2(B). (c) The second order results reflect the passing of the data through the temporal weighting function twice

on times of dominant activity. Dominant activity becomes clearer as you use higher order temporal weighting functions. This process only highlights the temporal locations of dense activity. The measure of this dominance should be viewed as being relative to surrounding temporal activity and not as an absolute measure.

Nonetheless, theses results are preliminary and the parameters of the processing technique will need further tuning. Pulse 'i' in Figure 2(a) for example, indicates the time at which a phone text message was made. Although the sensor detected this activity, when the power is averaged into one second blocks, the power level falls below that detectable by the process. Results in Fig. 4(c) show a high temporal peak at around time 13:39 from sensor one while sensor two's weighted results did not. The peak may have been generated by a mobile call close to the position of the sensor. This effect can be mitigated or further highlighted when taking into account information from a collaborative sensor network.

The analysis of RSSI data using temporal weighting has several positive features;

- Tuning of the parameters of the Gaussian weighting, (spread, weights, amplification) can highlight different characterises within the same RSSI data set

- Gaussian processes are known for being computationally intensive, but the preceding windowing, significantly reduces computational requirements

- Although not applied here, multidimensional data can be easily incorporated into the Gaussian weighting function

- Temporal spikes in activity can be identified easily

\section{Conclusions}

Preliminary findings suggest that monitoring cumulative receiver signal strength measurements (RSSI) of mobile phone signals can be a valuable tool in gathering information for mobile usage geographical mapping. There are both advantages and disadvantages to using cumulative RSSI data. However, given the capability of the metric to capture diverse mobile device activity without involving mobile network operators or intruding on the privacy of the general public, the flexibility far outweighs the difficulties in its reliable collection.

This approach could also be used to complement traditional techniques of mobile usage mapping. One could use the network operator data, if available, to model the dynamics of a city or town while RSSI data could be used to observe the dynamics of specific buildings or local regions within that urban environment.

Nonetheless, our research is still in its preliminary test stages, so additional validation of the results will have to be carried out. For this purpose, a mobile sensor network aimed at the collection of client-side mobile phone cumulative RSSI data is under construction. This sensor network will first be distributed throughout the north campus of NUI Maynooth with a view to expand into the nearby south campus and town of Maynooth, in the longer term. This project will offer us an opportunity to understand some of the dynamics relating to university student life. Moreover, by focusing on temporal and spatial patterns of mobile phone activity to give insight on how we interact with our local environment.

We hope to address such questions as how are buildings really used on campus, how do we determine where people can be found as opposed to where they pass through and how we can identify and localise local events as they occur in time and space. The answers to these questions would pave the way for a number of interesting applications. We could produce both indoor and outdoor maps showing the flow of people in real time around the campus. Such a map could be used by emergency services for rapid aid deployment in times of disasters or by university planning authorities.

\section{AcKNOWLEDGEMENTS}

Research presented in this paper was funded by ESRI Ireland in partnership with the Irish research Council for Science, Engineering and Technology under their Embark Initiative and by a Strategic Research Cluster grant (07/SRC/I1168) by Science Foundation Ireland under the National Development Plan. The authors gratefully acknowledge this support.

\section{REFERENCES}

[1] I. C. Regulator, "Quarterly report," COMREG, Tech. Rep., March 2009. 
[2] I. C. S. Office, "Population and migration estimates," CSO, Tech. Rep., April 2008.

[3] C. Ratti, S. Williams, D. Frenchman, and R. Pulselli, "Mobile landscapes: using location data from cell phones for urban analysis," Environment and Planning B: Planning and Design, vol. 33, no. 5, pp. 727-748, 2006.

[4] J. Reades, F. Calabrese, A. Sevtsuk, and C. Ratti, "Cellular census: Explorations in urban data collection," IEEE Pervasive Computing, vol. 6, no. 3, pp. 30-38, 2007.

[5] H. Urkowitz, "Energy detection of unknown," PROCEEDINGS OF THE IEEE, vol. 55, no. 4, pp. 30-38, April 1967.

[6] K. Srinivasan and P. Levis, "Rssi is under appreciated," 2006.

[7] C. Ratti, A. Sevtsuk, S. Huang, and R. Pailer, "Mobile landscapes: Graz in real time," pp. 2830, 2005.

[8] N. Eagle, A. Pentland, and D. Lazer, "Inferring social network structure using mobile phone data," Proc. of National Academy of Sciences, 2006.

[9] R. Wu, Y. Lee, H. Tseng, Y. Jan, and M. Chuang, "Study of characteristics of rssi signal," pp. 1-3, 2008.

[10] T. Stoyanova, F. Kerasiotis, A. Prayati, and G. Papadopoulos, "Evaluation of impact factors on rss accuracy for localization and tracking applications," pp. 9-16, 2007.

[11] S. Haykin, "Cognitive radio: brain-empowered wireless communications," IEEE Journal on Selected Areas in Communications, vol. 23, no. 2, pp. 201-220, 2005.

[12] W. L. I.F. Akyildiz, M. Vuran, and S. Mohanty, "Next generation/dynamic spectrum access/cognitive radio wireless networks: A survey," Computer Networks, vol. 50, no. 13, pp. 2127-2159, 2006.

[13] A. Ghasemi and E. Sousa, "Collaborative spectrum sensing for opportunistic access in fading environments," pp. 131-136, 2005.

[14] X. Huang, N. Han, G. Zheng, S. Sohn, and J. Kim, "Weighted-collaborative spectrum sensing in cognitive radio," pp. 110-114, 2007. 